\title{
Estimation of the Lag Time Between Detrusor Pressure- and Flow Rate-Signals
}

\author{
Ries Kranse, Ron van Mastrigt, and Ruud Bosch \\ Department of Urology-Urodynamics, Erasmus University, Rotterdam, The Netherlands
}

\begin{abstract}
In a urodynamic measurement setup there is a considerable spatial separation between the uroflowmeter and the location where the detrusor pressure is measured. Therefore, a ${ }^{*}$ time shift" (or lag time correction) has to be applied to one of these signals in order to align related samples in studies where pressure and flow rate are considered simultaneously (e.g. . assessment of bladder contractility or bladder outlet resistance).

Currently, a heuristic value for this time shift of $0.8 \mathrm{~s}$ is applied. In this article, we present a method to estimate the lag time directly from the measurements.

Using this method we have found, amongst others, that the mean lag time in our clinic is $0.6 \mathrm{~s}$ for males, $0.4 \mathrm{~s}$ for females voiding in sitting position, and $1.1 \mathrm{~s}$ for females voiding in standing position using a special receptacle in video urodynamics. Furthermore, we found that sphincter/urethral activity during voiding (which causes a drop in flow rate and an accompanying increase in detrusor pressure) is associated (on average) with shorter lag times than straining (when a positive pressure rise accompanies an increase in flow rate). Additionally strong evidence is provided that lag time correction is not a major source of error in urodynamics. 1995 Wiley-Liss. Inc.
\end{abstract}

Key words: cross correlation, pressure-flow, urodynamics

\section{INTRODUCTION}

In urodynamics, physical signals are measured in order to derive objective measures of the function of the lower urinary tract. These signals are, amongst others, the urinary flow rate and the detrusor pressure defined as the difference between the pressure measured in the bladder (the intravesical pressure) and the rectal pressure, which is considered equivalent to the abdominal pressure. In a urodynamic setup, the detrusor pressure is thought to originate from the bladder, whereas the flow rate is measured by a flowmeter that is separated by a distance of $15-60 \mathrm{~cm}$ (dependent on the specific type of measurement) from the external meatus of the patient. Therefore, there is a delay time between the measured flow rate signal and the measured detrusor pressure signal.

A simultaneous study of detrusor pressure and flow rate signals allows estima-

Received for publication June 23, 1994; accepted September 7, 1994.

Address reprint requests to Ries Kranse, Department of Urology, Room EE 1630, Erasmus University Rotterdam, P.O. Box 1738, 3000 DR Rotterdam, The Netherlands. 
tion of bladder outlet resistance [Griffiths et al., 1989; Schäfer, 1985; Spångberg et al., 1989; van Mastrigt and Kranse, 1993] and bladder contractility [Griffiths et al., 1986; van Mastrigt and Rollema, 1992], two properties that aid the physician in the differential diagnosis of voiding dysfunction and the choice of an adequate treatment modality [Rollema and van Mastrigt, 1992]. In such a study, related samples in pressure and flow rate signals have to be aligned by applying a time shift. Values of approximately I s [Griffiths, 1980] have been suggested. For our setup a time shift of $0.8 \mathrm{~s}$ has been in use. Possible values up to $5 \mathrm{~s}$ have been reported [Schäfer et al., 1990].

So far no systematic procedure has been published that allows determination of the lag time in a specific measurement setup. In a pilot study [Kranse and van Mastrigt, 1990] we presented a first attempt to estimate the lag time directly from the detrusor pressure and flow rate signals. The method used, however, can be criticized. In this article we present a superior method which enables the estimation of the average time shift that has to be applied to align detrusor pressure and flow rate signals in a specific measurement setup. It was applied to 260 measurements conducted in both males and females. The latter voided in two different positions. The different lag times involved were studied and the consequences for modelling of the urinary tract are discussed.

\section{MATERIALS AND METHODS}

Two hundred and sixty urodynamic measurements in 56 male and 74 female patients ( 30 female patients underwent video urodynamics, 44 underwent a standard urodynamic investigation) were studied ( 2 consecutive measurements for each patient). For all patients the filled volume, flow rate, abdominal $\left(\mathrm{p}_{\mathrm{athd}}\right)$ and intravesical pressures $\left(p_{v e s}\right)$ and a low pass filtered $(5 \mathrm{~Hz})$ rectified pelvic floor EMG were measured. All signals were sampled at a $10 \mathrm{~Hz}$ sampling rate (after $5 \mathrm{~Hz}$ analog low pass anti-aliasing filtering) and stored in a personal computer. The detrusor pressure was calculated as $p_{\text {ves }}-p_{\text {abd }}$. Men voided in sitting or standing position. The position of the funnel of the flowmeter was adjusted in such a way that the distance to the meatus for both situations was comparable. Women voided in standing (video urodynamics, 60 measurements) or sitting position (standard urodynamics, 88 measurements). In standing position a special hand-held funnel was used to guide the urine to the flowmeter (length of the device approximately $50 \mathrm{~cm}$ ). The flow rate signal was measured by means of a Dantec rotating disk flowmeter (Urodyn 1000), pressure signals were measured by means of disposable pressure transducers connected to water-filled catheters.

The voiding phase of the detrusor pressure and flow rate signals was selected for processing. To these signal parts a mathematical procedure was applied that is basically comparable to the calculation of the cross correlation function as was done in a previous study [Kranse and van Mastrigt, 1990]. In the present procedure an additional pre-processing step (called pre-whitening) was applied to the signals. The details of the method are explained in appendix A. Figure 1 gives a graphical overview. A cross correlation function is derived by first calculating the correlation coefficient between two signals. Then one of the signals is shifted by one sample and the correlation coefficient is calculated again. This procedure is repeated for all possible time shifts. The correlation coefficients calculated in this way are presented 

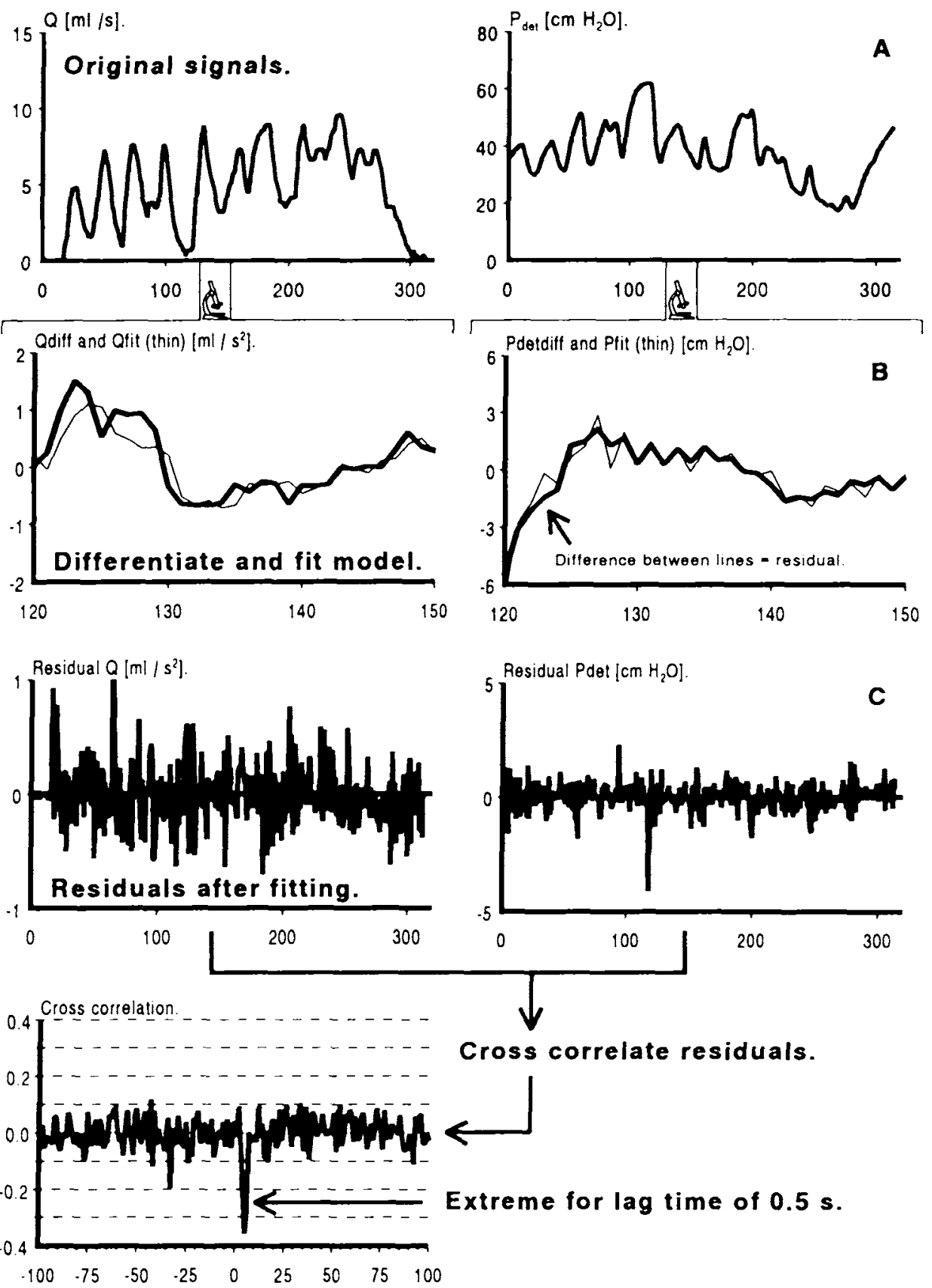

Time (In samples) on horizontal axes, 1 sample $=0.1$

Fig. 1. Overview of the signal processing involved in the estimation of the lag time between detrusor pressure and flow rate signals. The lag time between these signals is caused by the distance that separates the locations where these signals are measured in a urodynamic setup. A shows the original signals, B shows small episodes of the differentiated original signals in conjunction with the response of the fitted autoregressive models. In $\mathbf{C}$ the residuals that result from the subtraction of the fitted models from the differentiated original signals are depicted. The sample cross correlation function is obtained by "cross correlating" these two residuals. 
as a function of the time shift applied. Usually time shifts are applied in two directions; in our specific application negative time shifts correspond to physical impossibilities or non-causal events, i.e., variations in flow rate can never be measured before the associated pressure variations. Loosely speaking, a correlation coefficient measures the degree of likeliness with respect to mutual variations between two signals. Therefore, the calculated cross correlation function can be expected to attain an extreme value for the time shift that causes a "best match" between common signal variations. This time shift equals the lag time caused by the distance that separates flowmeter and pressure transducer. Common signal variations can be positively or negatively correlated and thus positive and negative peaks can occur in the cross correlation function. An interpretation of the polarity of the extreme in urodynamics is deferred to the Discussion section of this article.

The position (i.e., the lag time), the statistical level of significance or $P$ value (see Appendix A), and the polarity of the extreme of the first 100 time-shifts in positive and negative direction were determined from the calculated cross correlation functions of all measurements.

In order to obtain an average lag time estimation, the absolute values of the correlation functions for all male patients, all female patients who underwent standard urodynamics, and all female patients who underwent video urodynamics were summed. In the summed functions information on the type of extreme (positive or negative) is lost and therefore the absolute values of the negative and positive parts of the sample cross correlation functions were also summed separately.

As a first attempt towards quantifying the clinical significance of applying a correct time shift, values for the detrusor pressure at maximum flow rate $(\mathrm{p}(\mathrm{Qmax}))$, the detrusor opening pressure ( $p_{o p}$ ] [Abrams et al., 1988] (a flow threshold of $1 \mathrm{ml} / \mathrm{s}$ was used), and the detrusor closure pressure ( $\mathrm{p}_{\mathrm{cl}}$, defined in analogy to the detrusor opening pressure) were determined with and without applying the appropriate time shift (i.e., $0.6 \mathrm{~s}$ for males, $0.4 \mathrm{~s}$ for females in standard urodynamics, and $1.1 \mathrm{~s}$ for females in video urodynamics). For each parameter studied, the absolute value of the difference (between values derived from lag time corrected and uncorrected data) was calculated for each measurement. In this way separate estimates of the error caused by omission of lag time correction were obtained for the first and second measurement in each patient. Secondly for each parameter studied the absolute value of the difference between the parameters obtained in the two consecutive measurements in each patient was calculated (the inter measurement variation) in two ways (with and without lag time correction applied). The intra and inter measurement parametervariations thus obtained were compared by means of the Mann Whitney U-test.

\section{RESULTS}

Figure 2 shows an example of a measurement with a negative extreme in the cross correlation function. The cross correlation function shows an estimated lag time of $0.4 \mathrm{~s}$. Application of this time shift to a small section of the measured signals is shown in Figure 3. Figures 4 and 5 show similar graphs of a measurement with a very significant positive extreme in the cross correlation function.

In 47 of the 112 measurements in male patients, a significant extreme (either positive or negative, $P \leq 0.001$ ) was found in the cross correlation function. In 37 cases this peak was located in the causal part of the sample cross correlation function, 

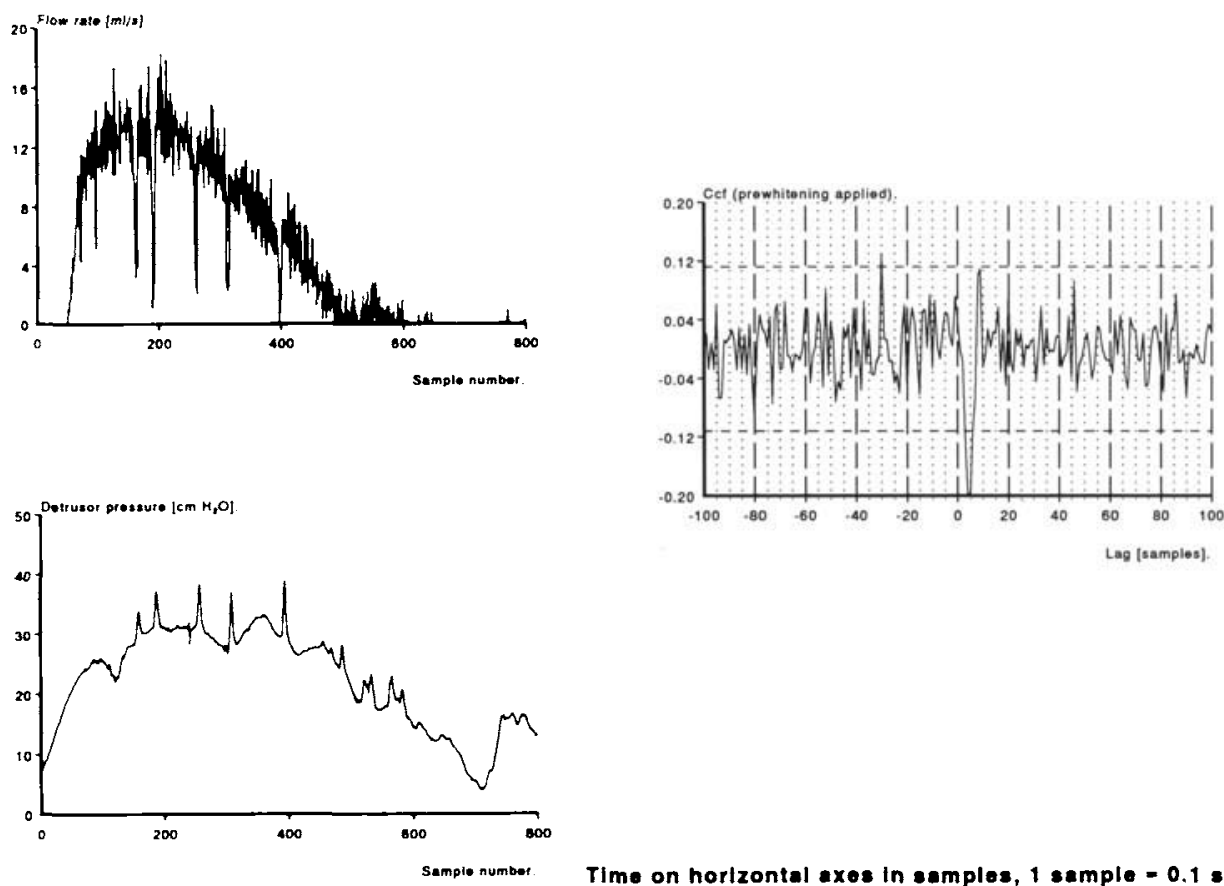

Fig. 2. Flow rate signal, detrusor pressure signal, and cross correlation function of a measurement with a negative extreme. Such a negative extreme implies that variations in flow rate and detrusor pressure signals have opposite polarities. These variations are probably related to urethral or sphincter activity.

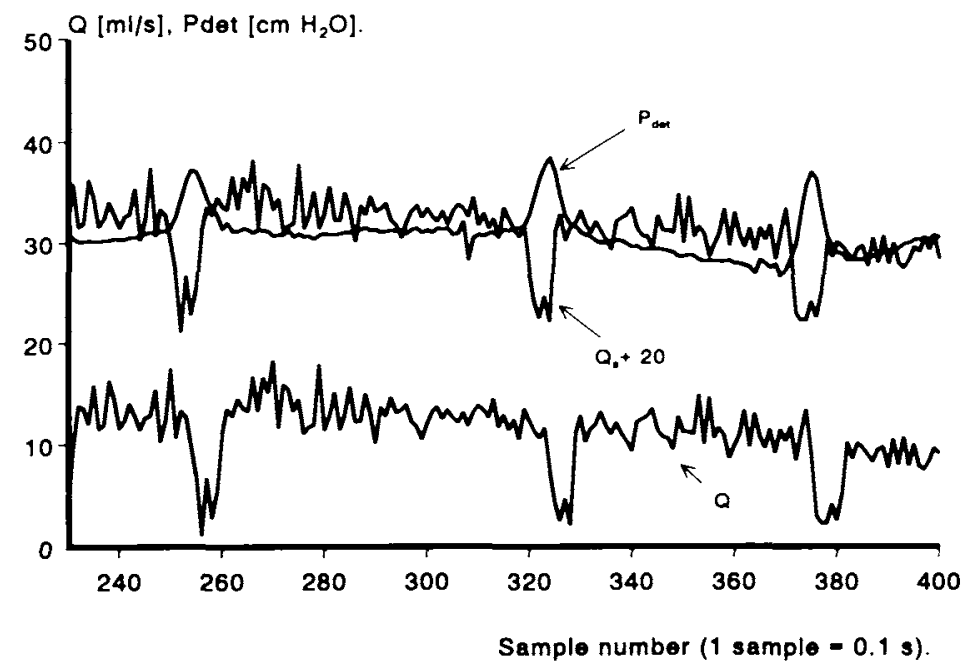

Fig. 3. Application of the lag time estimated from the cross correlation function in Figure 2 (of $0.4 \mathrm{~s}$ ) to a small section of it. The lag time corrected flow rate was shifted upwards to facilitate the comparison with associated pressure variations. 

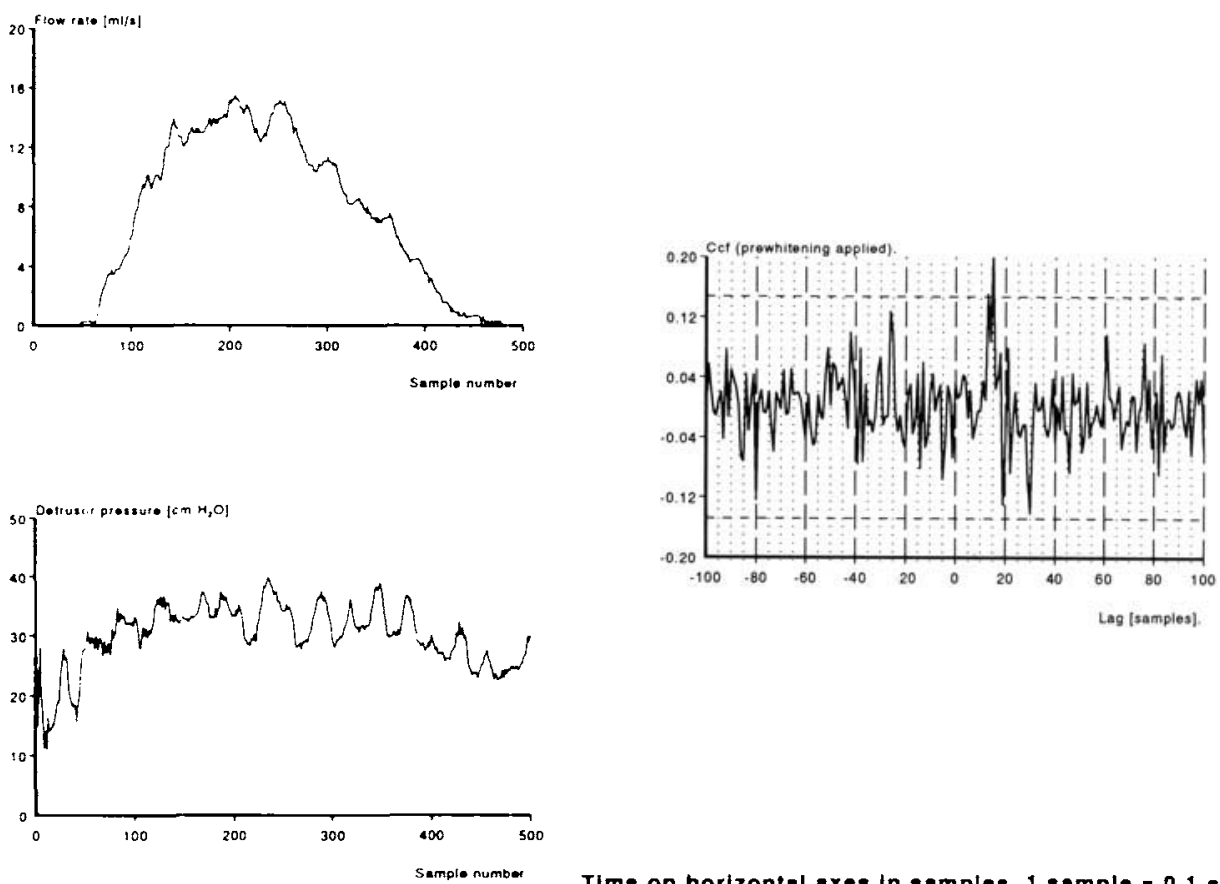

Fig. 4. An example of a measurement with positively correlated signal variations (note the positive peak in the cross correlation function). The shown signal variations are very likely related to straining.

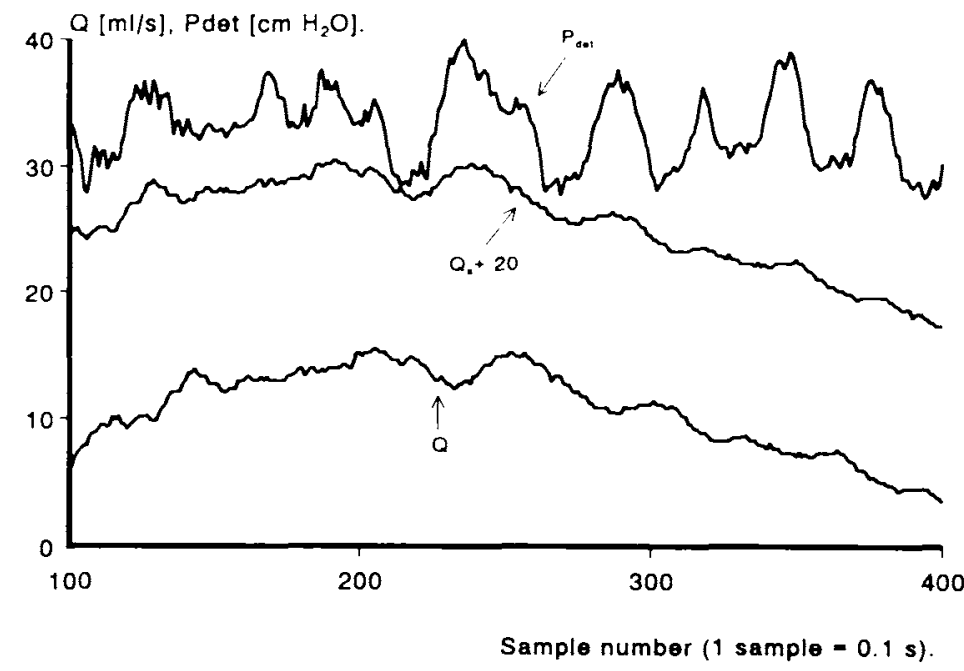

Fig. 5. Application of the estimated lag time (of $1.5 \mathrm{~s}$ ) to a small section of Figure 4. As in Figure 3 the lag time corrected flow was shifted upwards.

i.e., in the part that can be explained on a physical basis (see Materials and Methods). Seven measurements were found with a peak with $P<0.00000 \mathrm{I}$, the associated lag times were respectively $4,6,6,8,8,10$, and 12 samples. 

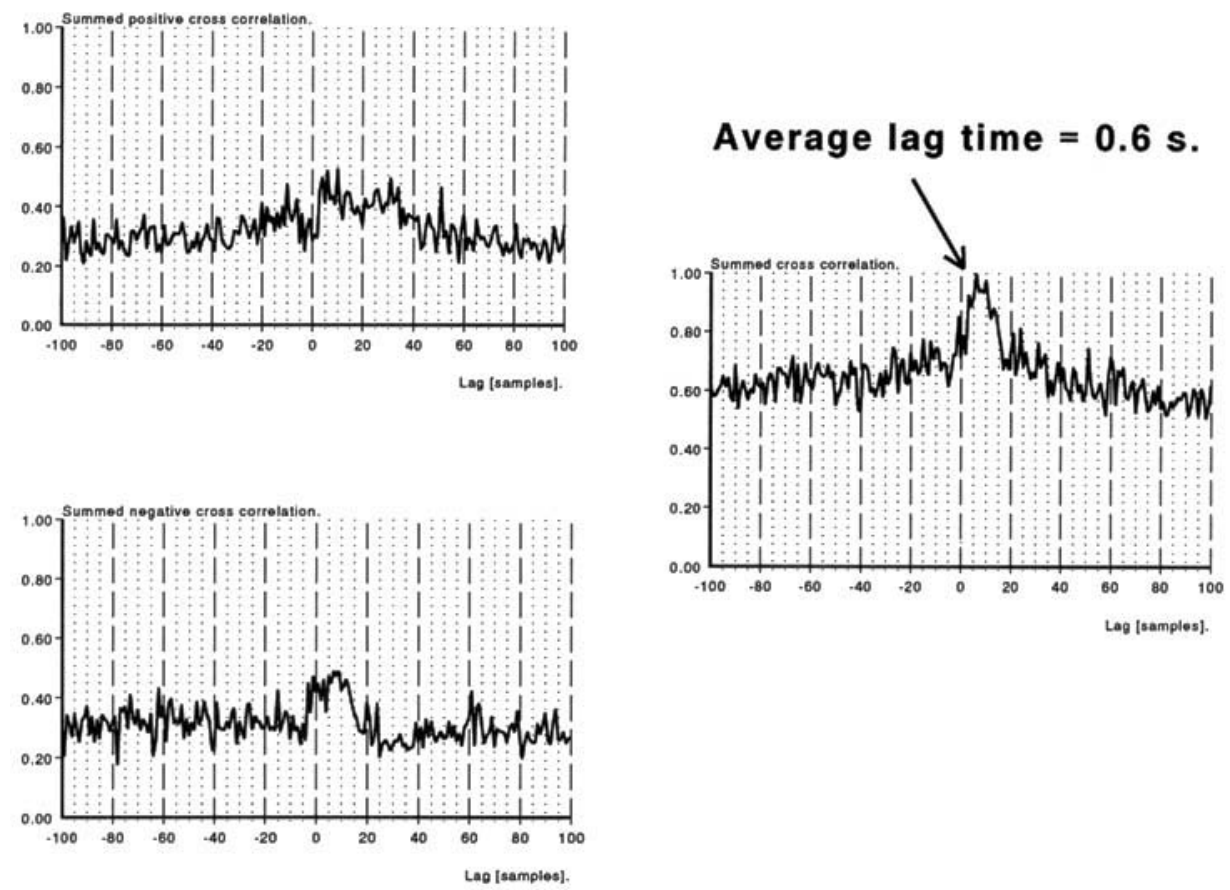

Tlme on horizontal axes in samples, 1 sample -0.1 ,

Fig. 6. Summed cross correlation and separately summed positive and negative cross comelation functions for male patients. The extreme in the summed cross correlation function represents an average lag time in male patients of $0.6 \mathrm{~s}$.

In the measurements in female patients, 59 significant extremes were found, 18 of which occurred for negative lags (non-causal). Fourteen measurements had a peak with $P<0.000001$, the associated lag times were respectively $-79,-21,2,4,4$, $5,5,7,9,9,9,11,15$, and 22 samples.

Figure 6 shows the cumulative cross correlation function for the male patients together with the separate cumulative cross correlation functions for positive and negative excursions only. The lag time for the overall extremes was $0.6 \mathrm{~s}$. Figure 7 gives the same graphs for female patients who underwent standard urodynamics and Figure 8 gives the results obtained for female patients who underwent video urodynamics. The overall lags for these two measurement types in women were 0.4 and 1.1 s, respectively.

Table $I$ compares the errors in $p(Q \max ), p_{o p}$ and $p_{c l}$ when lag time correction was omitted with the differences between two successive measurements in the same patient. The former variation was significantly smaller than the latter one (in Table I, of all possible comparisons, only the least significant $P$ values are listed). The influence of the receptacle used in video urodynamics can be observed from the higher inter measurement parameter variability in this group.

\section{DISCUSSION}

In the current study, the lag time between the detrusor pressure and flow rate signals in a urodynamic measurement setup was estimated by means of a statistical 

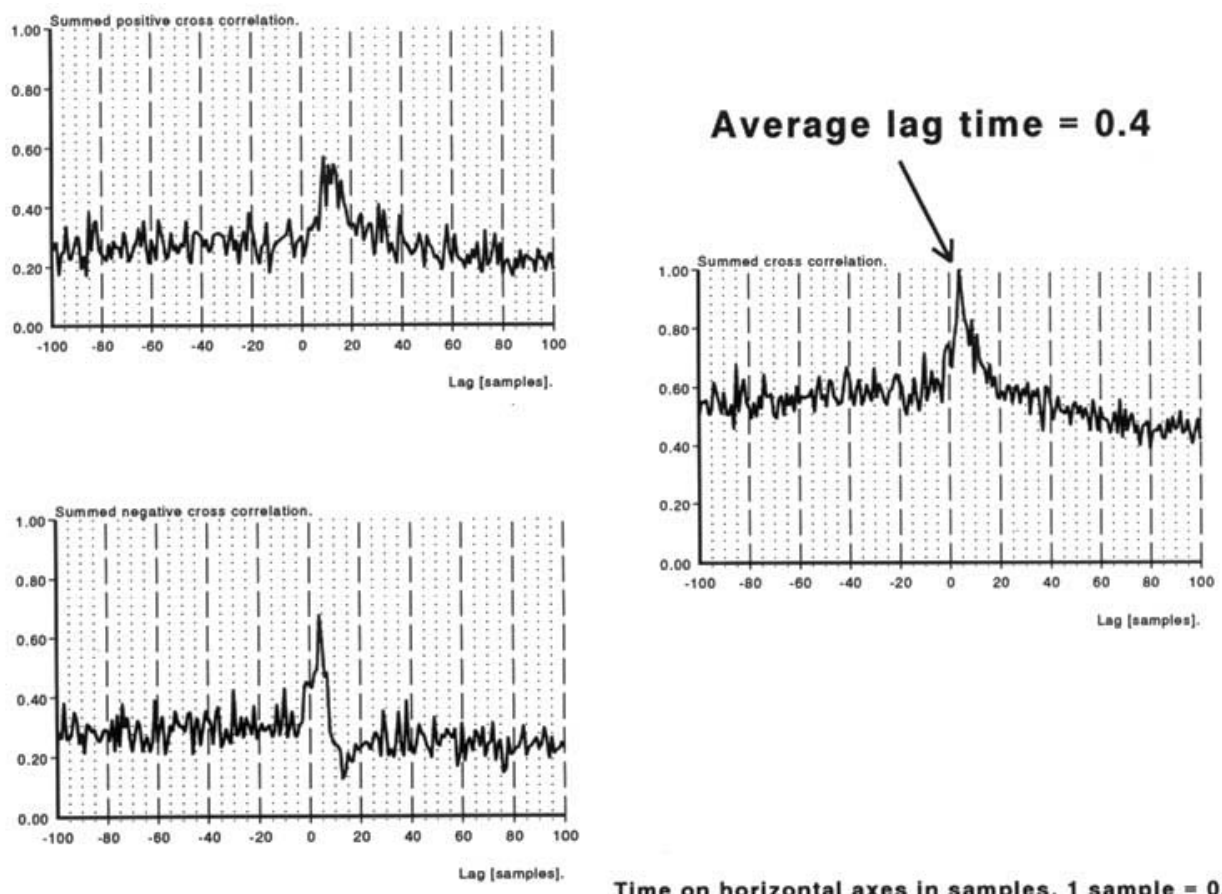

Time on horizontal axes in samples, 1 sample $=0.1 \mathrm{~s}$

Fig. 7. Summed cross correlation and separately summed positive and negative cross correlation functions for female patients that underwent standard urodynamics (average lag time $=0.4 \mathrm{~s}$ ).

procedure based on the cross correlation function. Related variations in detrusor pressure and flow rate signals are shifted in time as a consequence of the distance that separates the flowmeter from the location of pressure measurement. When common signal variations occur the lag time causes a statistically significant peak in the calculated cross correlation function.

We found only 106 (47 for males +59 for females) significant $(P \leq 0.001)$ extremes in 260 measurements studied, implying that for the majority of measurements $(154=260-106)$ no lag time estimation was possible. This can be explained in two ways. The most probable explanation in our view is that in these measurements related variations in the detrusor pressure and flow rate signals were absent. An alternative explanation is that the lag time varied drastically during these measurements so that none of the "fluctuating lag times" present gave rise to a significant peak in the cross correlation function. Such a variation might possibly result from variations in the position of the point of impact of the urinary stream on the flow meter funnel.

Twenty-eight (10 for males and 18 for females) significant peaks occurred for negative delays (i.e., non-causal events, see Materials and Methods) which correspond to physical impossibilities. These numbers can be explained on the basis of the $P$ value used in the definition of a significant extreme $(P \leq 0.001)$. We searched for significant extremes in 260 cross correlation functions. The search was limited to 201 points (i.e., no lag, 100 non-causal lag times, and 100 causal lag times) so that 

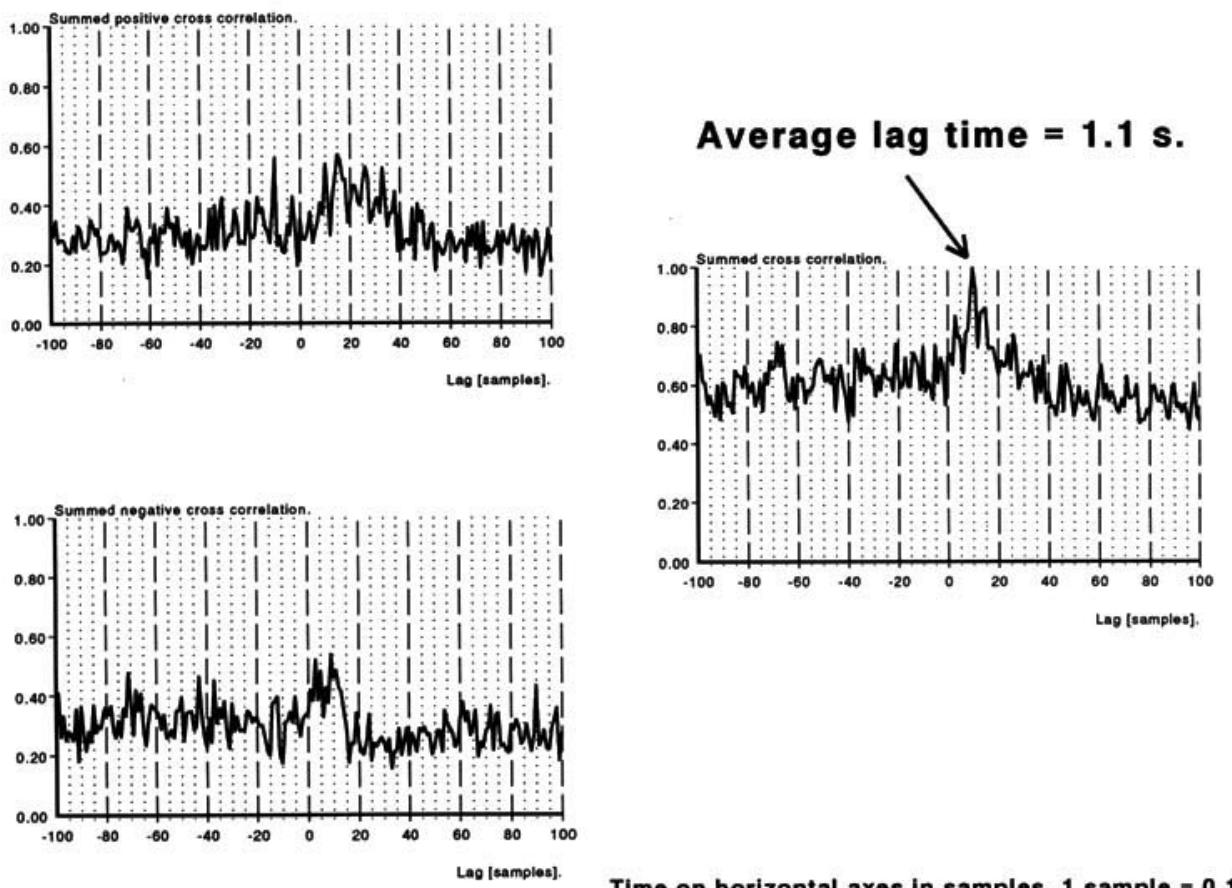

Time on horizontal axes in samples, 1 sample $=0.1 \mathrm{~s}$

Fig. 8. Summed cross correlation and separately summed positive and negative cross correlation functions for female patients that underwent video urodynamics (average lag time $=1.1 \mathrm{~s}$ ).

$201 \times 260=52,260$ cross correlation entries were inspected. Therefore, $0.001 \times$ $52,260=52$ spurious extremes could be expected. Half of these should occur in the causal part of the cross correlation function which compares favourably with 28 occurrences found. The previous argument also leads to the conclusion that only 106 $-2 \times 26=54$ of the extremes detected from the total of 260 measurements processed (roughly $20 \%$ ) correspond to common signal variations that are shifted in time. Interpretation of a cross correlation function of an individual measurement is further complicated by the fact that in some rare cases ( 2 measurements, both in females, out of 260) common signal variations were present that cannot be causally related but that did give rise to a very significant extreme in the calculated cross correlation function $(P \leq 0.000001)$. This does not disqualify the method used; visual inspection of the measurements showed common artifacts in both signals.

In conclusion, we can state that because of the difficulties with the interpretation of extremes (i.e., is an extreme spurious or not?) the practical applicability of the method used in individual measurements is limited.

Summation of the absolute values of the sample cross correlation functions in the whole group of patients tested, however, yielded a clear impression of the average lag time for the specific measurement setup in males, females who underwent standard urodynamics and females who underwent video urodynamics. Figure 6 shows that the summed cross correlation function for males has an extreme for a lag time of $0.6 \mathrm{~s}$ and it is obvious that most of the extremes in this summed cross correlation can be related to negatively correlated variations in detrusor pressure and flow rate signals 
TABLE I. The Error in Detrusor Pressure at Maximum Flow Rate (p(Qmax)), the Opening Pressure $\left(p_{o p}\right)$, and the Closure Pressure $\left(p_{c l}\right)$ When Lag Time Correction Was Omitted Compared to the Difference Between Two Successive Measurements in the Same Patient*

\begin{tabular}{|c|c|c|c|c|c|}
\hline \multirow[b]{2}{*}{ Patients } & \multirow[b]{2}{*}{$\begin{array}{c}\text { Parameter } \\
\text { studied }\end{array}$} & \multicolumn{2}{|c|}{$\begin{array}{c}\text { Error caused by } \\
\text { omission of lag time } \\
\text { correction }\left[\mathrm{cm} \mathrm{H}_{2} \mathrm{O}\right]\end{array}$} & \multicolumn{2}{|c|}{$\begin{array}{c}\text { Difference in } \\
\text { parameter studied } \\
\text { between successive } \\
\text { measurements in } \\
\text { one patient } \\
{\left[\mathrm{cm} \mathrm{H}_{2} \mathrm{O}\right]}\end{array}$} \\
\hline & & $\begin{array}{c}\text { Measure- } \\
\text { ment } 1\end{array}$ & $\begin{array}{c}\text { Measure- } \\
\text { ment } 2\end{array}$ & $\begin{array}{c}\text { No time shift } \\
\text { applied }\end{array}$ & $\begin{array}{c}\text { Time shift } \\
\text { applied }\end{array}$ \\
\hline \multirow[t]{3}{*}{ Males, $0.6 \mathrm{~s}$} & $\mathrm{p}(\mathrm{Qmax})$ & $1.6^{* *}$ & $2.1^{* *}$ & 9.1 & 8.7 \\
\hline & $p_{o p}$ & $2.0^{* *}$ & $2.1^{* *}$ & 13.0 & 13.2 \\
\hline & $\mathrm{Pcl}_{\mathrm{cl}}$ & $3.7^{* *}$ & $3.6^{* *}$ & 19.0 & 19.8 \\
\hline \multirow{3}{*}{$\begin{array}{l}\text { Females, } \\
\text { standard urodynamics } \\
\text { lag time } 0.4 \mathrm{~s}\end{array}$} & $\mathrm{p}(\mathrm{Qmax})$ & $1.2^{* *}$ & $1.0^{* *}$ & 6.2 & 6.4 \\
\hline & $p_{o p}$ & $2.3 * *$ & $1.5^{* *}$ & 9.5 & 9.1 \\
\hline & $\mathrm{p}_{\mathrm{cl}}$ & $2.4^{* *}$ & $2.2^{* *}$ & 11.5 & 9.2 \\
\hline \multirow{3}{*}{$\begin{array}{l}\text { Females, } \\
\text { video urodynamics } \\
\text { lag time } 1.1 \mathrm{~s}\end{array}$} & $\mathrm{p}(\mathrm{Qmax})$ & $3.6(P=0.02)$ & $2.0(P=0.007)$ & 7.9 & 6.8 \\
\hline & $\mathrm{p}_{\mathrm{op}}$ & $4.6(P=0.003)$ & $3.7(P=0.006)$ & 9.2 & 9.0 \\
\hline & $p_{\mathrm{c}}$ & $6.0(P=0.007)$ & $3.0(P=0.002)$ & 15.3 & 12.0 \\
\hline
\end{tabular}

*Of all possible comparisons the least significant p-values are listed.

$* * P<0.0001$.

(as can be observed by studying the separately summed positive and negative cross correlation functions). Negative correlations can be associated with urethral or sphincter activity (a decrease in flow rate accompanied by an increase in detrusor pressure, see the example in Fig. 2). Comparison of the graphs in Figure 7 shows that the extreme for females who underwent standard urodynamics occurred for a delay of $0.4 \mathrm{~s}$. In these measurements positive as well as negative correlations were found. As the peak in the separately summed positive correlations is positioned to the right (at $0.9 \mathrm{~s}$ ) of the peak in the separately summed negative correlations (at $0.4 \mathrm{~s}$ ), we conclude that positive correlations (very likely related to straining where an increase in detrusor pressure is accompanied by an increase in flow rate) are related to longer lag times. In addition lag times caused by positively correlated events (straining) seem to vary over a wider range of values than lag times caused by negatively correlated events. This latter observation can be explained by the fact that the degree of straining can be varied whereas urethral or sphincter activity has a more or less on-off character. The extreme of the cumulative cross correlation function for video urodynamic measurements (females who voided in a standing position using a handheld funnel) occurred for a lag time of $1.1 \mathrm{~s}$. Again positive and negative lags were found and again it seems that positive correlations are "slower" than negative ones (it seems from Fig. 6 that this is probably also true for males). The fact that negative correlations occur at smaller lag times than positive ones in all patient groups can be explained by the observation that urethral/sphincter contraction during a bladder contraction usually occurs in that part of micturition where the fluid velocity is relatively high. In contrast straining usually occurs when voiding is troublesome (and probably at low fluid velocities).

Inspection of Figures 6,7 , and 8 leads to the conclusion that in our measure- 
ment setup lag times are (approximately) limited to the interval of $0.2-2 \mathrm{~s}$. Comparison of Figure 7 and 8 shows that voiding through the hand-held funnel (as used for video urodynamics) leads to an extra signal delay of $0.7 \mathrm{~s}$ and to extra noise.

The fact that the error in $\mathrm{p}(\mathrm{Qmax}), \mathrm{p}_{\mathrm{op}}$, and $\mathrm{p}_{\mathrm{cl}}$ caused by omission of lag time correction was significantly smaller than the difference between two successive measurements in one patient (Table I) indicates that the clinical relevance of optimal lag time correction is probably limited. Although the parameters mentioned are not the only urodynamic parameters that might depend on lag time we think it is very unlikely that other parameters or functions (e.g., bladder contractility as a function of bladder volume) are more sensitive to optimal lag time correction.

In conclusion, it can be stated that the method presented in this study is very suitable to assess the average lag time between detrusor pressure and flow rate signals in a specific urodynamic measurement setup. Estimation of these average lag time values enables the application of optimal time shifts in the calculation of bladder contractility and bladder outlet resistance parameters in males and females (in standard and video urodynamics). Based on our results we suggest to use lag time corrections of $0.4 \mathrm{~s}$ (for sitting voiding) and $1.1 \mathrm{~s}$ (for standing voiding using a hand-held receptacle) when studying measurements in females and of $0.6 \mathrm{~s}$ when studying measurements in males (if a comparable setup is used).

Application of the method further showed that pressure and flow rate variations associated with urethral or sphincter activity have smaller lag times than signal variations associated with straining and that the range of lag times associated with signal variations caused by straining is wider than that for signal variations caused by urethral or sphincter activity.

When no lag time correction was applied the error in the detrusor pressure at maximum flow rate amounted to $3.6 \mathrm{~cm} \mathrm{H}_{2} \mathrm{O}$ in the worst case (females voiding in a standing position through a hand held funnel). When compared to the observation that the average difference between two consecutive measurements in the same group amounted to $6.8 \mathrm{~cm} \mathrm{H}_{2} \mathrm{O}$ this strongly suggests that lag time correction is not a major source of error in urodynamics.

\section{REFERENCES}

Abrams P, Blaivas JG, Stanton LS. Anderson JT (1988): Standardisation of terminology of lower urinary tract function. Neurourol Urodyn 7:4()3-427.

Chatfield C (1989): "The Analysis of Time Series." London: Chapman \& Hall.

Griffiths DJ (1980): "Urodynamics: The Mechanics and Hydrodynamics of the Lower Urinary Tract. Medical Physics Handbooks 4." Bristol: Adam Hilger Ltd.

Griffiths DJ. Constantinou CE. van Mastrigt R (1986): Urinary bladder function and its control in normal females. Am J Physiol 251:R225-R230.

Griffiths JD, van Mastrigt R, Bosch R (1989): Quantification of urethral resistance and bladder function during voiding. with special reference to the effects of prostate size reduction on urethral obstruction due to benign prostatic hyperplasia. Neurourol Urodyn 8:17-27.

Jenkins GM, Watts DG (1968): "Spectral Analysis and Its Applications." San Francisco, CA: HoldenDay, Inc

Kranse M, van Mastrigt R (1990): Cross correlation of detrusor pressure and flow rate signals. Proceedings of the ICS. Neurourol Urodyn 9:387-388.

Rollema HJ, van Mastrigt R (1992): Improved indication and follow-up in trans urethral resection of the prostate (TUR) using the computer program CLIM. J Urol 148:111-116.

Schäfer W (1985): Urethral resistance? Urodynamic concepts of physiological and pathophysiological bladder outlet function during voiding. Neurourol Urodyn 4:|6|-201. 
Schäfer W, Langen PH, Thörner M (1990): The real pressure/flow-relation during obstructed voiding. Proceedings of the ICS. Neurourol Urodyn 9:423-424.

Spångberg A, Teriö H, Engberg E, Ask P (1989): Quantification of urethral function based on Griffiths' model of flow through elastic tubes. Neurourol Urodyn 8:29-52.

van Mastrigt R. Rollema HJ (1992): Prognostic value of bladder contractility in prostatectomy. J Urol 148:1856-1860.

van Mastrigt R, Kranse M (1993): Automated evaluation of urethral obstruction. Urology 42:216-224.

\section{APPENDIX A}

The theory used in this appendix is discussed in much greater detail in The Analysis of Time Series by C. Chatfield [Chatfield, 1989] and in Spectral Analysis and Its Applications by Jenkins and Watts [Jenkins and Watts, 1968].

The lag time estimation procedure applied to the detrusor pressure- and flow rate-signals comprised the following steps (see Fig. 1): first, the detrusor pressure and flow rate signals were detrended by differentiation (in this way slow trends were removed from the signals). Next, a statistical model was fitted to the data (an auto regressive model). The result of this process of differentiation and model fitting is depicted in row B of Figure 1. The residuals (which equal the difference between the fitted models and differentiated signals) can be described as white noise (see row $\mathrm{C}$ of Fig. 1). They, however, still contain common time shifted variations provided these were present in the original signals. Cross correlation of the residuals gave a function that attained an extreme value for the correct time-shift. Mathematically the calculation of the cross correlation function can be formulated as follows:

$$
r_{x y}(k)=\frac{c_{x y}(k)}{\sqrt{(\operatorname{stddev}(x) \operatorname{stddev}(y))}}
$$

where $k=-(N-1) . . N-1, r_{x y}(k)$ is the cross correlation for time shift $k, c_{x y}(k)$ is the cross covariance for time shift $k$, and stddev $(x) * \operatorname{stddev}(y)$ denotes the product of the standard deviations of the time series $x$ and $y$.

$\mathrm{C}_{\mathrm{xy}}(\mathrm{k})$ is defined by (2) and (3).

$$
c_{x y}(k)=\sum_{t=1}^{N-k}\left(x_{t}-\bar{x}\right)\left(y_{t+k}-\bar{y}\right) / N
$$

for $\mathrm{k}=0,1 \ldots \mathrm{N}-1$, and

$$
c_{x y}(k)=\sum_{t=1-k}^{N}\left(x_{t}-\bar{x}\right)\left(y_{t+k}-\bar{y}\right) / N
$$

for $\mathrm{k}=-1,-2, . .-(\mathrm{N}-1)$.

In formulae 2 and $3 x_{1}$ and $y_{1}$ denote the individual samples of time series $x$ and $y, N$ the total number of samples in the series, and $\bar{x}$ and $\bar{y}$ are the averages of the time series $x$ and $y$.

Extremes in this sample cross-correlation function can be interpreted in a statistical context if the two signals $x$ and $y$ are white noise processes. For two such uncorrelated white noise signals the expected value of the sample cross correlation function varies around a mean of zero with a standard deviation of $1 / \mathrm{sqrt}(\mathrm{N})(\mathrm{N}$ is the number of samples used in the calculation). Two adjacent terms of this sample cross 
correlation function are practically uncorrelated for large values of $\mathrm{N}$. Assuming that entries in the sample cross correlation function are distributed according to a normal distribution (which is reasonable for large values of $\mathrm{N}$ because they basically equal the sum of a large number of identically distributed random variables, see formulae 1,2 , and 3), a test for non-zero cross correlation can be used.

Computer simulation experiments indicated that lag times between two timeshifted series could be detected even when the amplitude of noise signals equalled 2.5 times the amplitude of the shifted signals.

All signal processing was done by means of the Matlab ${ }^{\mathrm{TM}}$ system identification package. The Matlab source code of the procedure outlined above can be obtained from the authors. 